\title{
DEVELOPMENT AND APPLICATION OF A PRODUCT MODEL FOR SHIELD TUNNELS
}

\author{
*Nobuyoshi Yabuki \\ Osaka University \\ 2-1 Yamadaoka, Suita \\ Osaka, Japan 565-0871 \\ (*Corresponding author: yabuki@see.eng.osaka-u.ac.jp) \\ Takashi Aruga \\ Conport Co., Ltd. \\ 5-20-14-201 Hinohonmachi, Hino \\ Tokyo, Japan 191-0011 \\ Hiroshi Furuya \\ Obayashi Corporation \\ 4-40 Shimokiyoto, Kiyose \\ Tokyo, Japan 204-8558
}




\title{
DEVELOPMENT AND APPLICATION OF A PRODUCT MODEL FOR SHIELD TUNNELS
}

\begin{abstract}
Building Information Modeling is a prevailing methodology in the building industry and half (by length) of the world's shield tunnels are in Japan. Accordingly, for shield tunnels from 2005 to 2007, we developed a product model called IFC-ShieldTunnel, which is based on Industry Foundation Classes (IFC), in order to meet the future demand for such a product model. IFC-ShieldTunnel was developed by adding new elements that are specific to shield tunnels and that are not included in the original IFC. The added elements include shafts, segments (A, B, and $\mathrm{K}$ types), waterproofing elements, segment joint elements, and ring joint elements. Furthermore, soil layers and void elements were created. Recently, we updated the product model by re-organizing the entities and correcting errors. We deployed the updated IFCShieldTunnel model in the construction of an actual shield tunnel in Tokyo for about a year from August 2010 in order to check its feasibility. In the verification process, first, segments were modeled using Autodesk Revit Structure 2011 and property data were assigned to each segment element. Next, a mapping between the original attributes of the entities of IFC and properties of IFC-ShieldTunnel segments was developed. Using this mapping and IFC's IfcBuildingElementProxy function, we were able to convert the IFC data from Revit Structure 2011 to IFC-ShieldTunnel automatically. Segment data were also converted from Revit Structure to Google SketchUp by IFC-ShieldTunnel. Furthermore, the construction scheduling and cost data were converted from Revit Structure to EXCEL for 4D and 5D analyses of construction. We interviewed engineers who used IFC-ShieldTunnel and received positive comments about the utilization of the product model. Overall, our test demonstrated the feasibility of IFC-ShieldTunnel. In future work, we plan to apply IFC-ShieldTunnel to more projects and test its practicality.
\end{abstract}

\section{KEYWORDS}

Tunnel, Product model, Interoperability, IFC, Schema

\section{INTRODUCTION}

Recently, new construction methods, such as Automated Machine Control and Automated Machine Guidance have been developed. These methods utilize advanced technologies, including Global Positioning System, various small sensors, and auto tracking Total Stations, and are used to increase efficiency and improve quality (Furuya \& Fujiyama, 2011). Building Information Modeling has also been adopted for building design and construction in a number of nations and it is significantly changing the conduct of business.

In new trends like these, 3D models play a core role. However, if a 3D model specification depends on a specific software package, the model data will lack interoperability and cannot be exchanged or shared with other software packages (Eastman, 1999). Thus, the Standard for the Exchange of Product (STEP) model data have been developed as ISO-10303. In the domain of buildings, Industry Foundation Classes (IFC), developed as a building project model by buildingSMART (previously IAI, International Alliance for Interoperability) became International Standard, ISO 16739 in 2013 (buildingSMART, 2013). In the infrastructure domain, LandXML has been used widely as a de facto standard for the representation of roads, although some nations have their own road data models, including TransXML, Okstra, and Japan's Standard for the Exchange of Road Centerline Data. However, for some infrastructure elements, such as bridges, there are no internationally accepted standards. (Although IFC-Bridge, which is an 
extension of IFC to represent bridges, has been developed by a collaboration between Japan and France (Yabuki et al., 2006), it has not yet been incorporated into IFC.) To address this lack of standards, the OpenINFRA consortium was founded in 2012 within buildingSMART International to establish IFC-based product models for infrastructure, such as roads, bridges, tunnels, and harbors.

IFC-ShieldTunnel was developed by extending IFC to standardize the data model for representing shield tunnels (Yabuki, 2008; Yabuki et al., 2007). However, this data model was not used in practice because no CAD vendor made a program for converting data between their own CAD representation and that of IFC-ShieldTunnel. Typically, software vendors wait to see whether ISO or nations will try to standardize the data model before making data conversion programs. However, if no CAD or software vendor makes any data conversion program, nobody will want to use the proposed data model. Therefore, we have updated the previous version of IFC-ShieldTunnel to expand its versatility by devising a data conversion method, and we have applied it to an actual shield tunnel construction project in Tokyo.

\section{PRODUCT MODELS}

A product model is a generalized data model, based on object-oriented concepts, to enable the interoperable sharing and exchanging of a product's data, such as geometric shape, attributes, and relationships, among heterogeneous software packages throughout the lifecycle of the product. When developing a product model, it is important to define clearly scope, terminology, relationships, etc. The basic elements of a product model are called entities. Entities can represent not only physical objects but also processes, humans, organizations, data, knowledge, and so on. Each entity's attributes, including dimensions, material, and specification, are defined. Entities may be divided into smaller components, which may be divided still further. Relationships between such components are called "part-of" relations. On the other hand, entities can be classified as belonging to specific groups: for example, an animal can be classified as a mammal, bird, reptile, amphibian, or fish. This kind of relationship is called an "is-a" relation. Other relationships, such as "supported-by" and "contact-to", can also be defined between entities.

\section{CONCEPTUAL PRODUCT MODEL OF SHIELD TUNNELS}

\section{Shield Tunnels}

Shield tunnels are usually constructed for infrastructure such as highways, subways, sewage systems, and causeways where the cut-and-cover tunneling method cannot be employed since there are structures or natural features, such as rivers, that cannot be removed, lying above the route. First, a shaft tunnel is excavated and parts of a tunnel boring machine are lowered down the shaft and assembled. A tunnel boring machine consists of a shield and trailing support mechanisms. The front end of the shield has a cutting wheel, followed by a chamber, behind which there is a set of hydraulic jacks for pushing the shield forward. A tunneling ring which consists of several precast concrete or steel segments is installed between the shield and the surrounding soil. The set of tunneling rings is called the primary lining. If necessary, a secondary lining, which is made of concrete, may be constructed.

\section{Development of the Conceptual Product Model of Shield Tunnels}

The data that need to be defined in product models can be summarized as $5 \mathrm{~W} 1 \mathrm{H}$ : when, who, where, what, why, and how. Thus, in the development process of a conceptual product model of shield tunnels, a Product class for representing What and Where, a Process class for When and How, an Organization class for Who, and a Measured Data and Knowledge class for Why were placed under the Root of all classes. Objects, such as members, components, facilities, ground layers, processes related to shield tunnel construction, concrete organizations and stakeholders, and various sets of data and knowledge were included by consulting various documents and by interviewing shield tunnel experts. In this way, a conceptual, hierarchical product model was developed for representing shield tunnels. 


\section{IMPLEMENTATION OF IFC-SHIELDTUNNEL}

The conceptual shield tunnel product model was implemented by extending IFC Version $2 \times 3$ (IFC2x3) and adding necessary classes that were not already defined in IFC, such as members specific to shield tunnels and underground layers. The product model was named IFC-ShieldTunnel following the pattern of IFC-Bridge. As mentioned before, the implementation process had two phases. The first phase was from 2005 to 2007, and the second was from 2009 to 2010. The following description of the implementation combines these two phases into one.

In IFC, all entities have "Ifc" at the start of their names. IfcObject exists as a subclass of IfcObjectDefinition, and IfcElement exists as a subclass of IfcObject. We defined IfcCivilEment as a subclass of IfcElement to represent members specific to civil infrastructure. Entities that are specific to shield tunnels were defined by adding IfcStElement (St stands for Shield Tunnel) as a subclass of IfcCivilElement, and entities such as IfcStShaftElement, IfcStTunnelElement, IfcStJointStructureElement, IfcStTemporaryFacility, and IfcStShieldMachineElement were defined as subclasses of IfcStElement, following the developed conceptual product model of shield tunnels. Figure 1 shows a part of the schema of IFC-ShieldTunnel. A rounded rectangle indicates that it has super- or subclasses which are not shown in the figure. Figure 2 shows components, such as A, B, and K segments, joints between segments, and secondary lining, and their corresponding IFC-ShieldTunnel classes.

Underground soil layers are represented by the "upper boundary surface" method. In this method, each soil upper boundary surface is defined by the name of the soil layer below it, and any point in any soil layer can be classified by looking up the name of the immediately overlying upper boundary surface (Figure 3). Each soil layer can be geometrically represented using a Triangulated Irregular Network and modeled by IfcStStratumElement, which is an immediate subclass of IfcElement. Groundwater can be represented by IfcStGroundwaterElement.

A tunnel is an underground cave made by an excavation in solid earth. Cave objects are represented by IfcStVoidElement in IFC-ShieldTunnel. The upper surface of the void element is the boundary surface of the void itself, and the lower surface is related to the lower soil layer, following the upper boundary surface method.

The schema of IFC-ShieldTunnel was written in the EXPRESS language and graphically represented using EXPRESS-G, following ISO-STEP. Then the schema was translated into ifcXML to facilitate computer programming. In 2007, a data conversion program was made to read an instance file written in ifcXML and to draw 3D models in AutoCAD using Visual Basic for Applications.

\section{APPLICATION OF IFC-SHIELDTUNNEL TO CONSTRUCTION}

The IFC-ShieldTunnel model was applied to an actual construction project from August 2010 to August 2011 to test the model and its interoperability. The site was a part of the tunnel from the Oi Junction to the Ohashi Junction of the Central Beltway Shinagawa Line in Tokyo, Japan. The shield tunnel consists of two parts: one going uptown (length: $550 \mathrm{~m}$ ) and the other one going downtown (length: $336 \mathrm{~m}$ ).

In this verification, we focused our attention on segments because segments are the main parts of a shield tunnel. For modeling the tunnel structures and terrain, Revit Structure 2011 and Civil 3D from Autodesk were used. Figure 4 shows a tunnel ring consisting of three types of segments: A, B and K. Each segment type has two subtypes, thus there are six kinds of segment in all. These segment models were laid out along the centerline using the 3D CAD software.

Next, a set of property data was assigned to the segment model using Revit Structure (Table 1).

The parameters of width $\mathrm{H}$ and curvature $\mathrm{A}$ were used as input data for the parametric design of each segment. Identification information (manufacturer, price, etc.) was defined as well. 


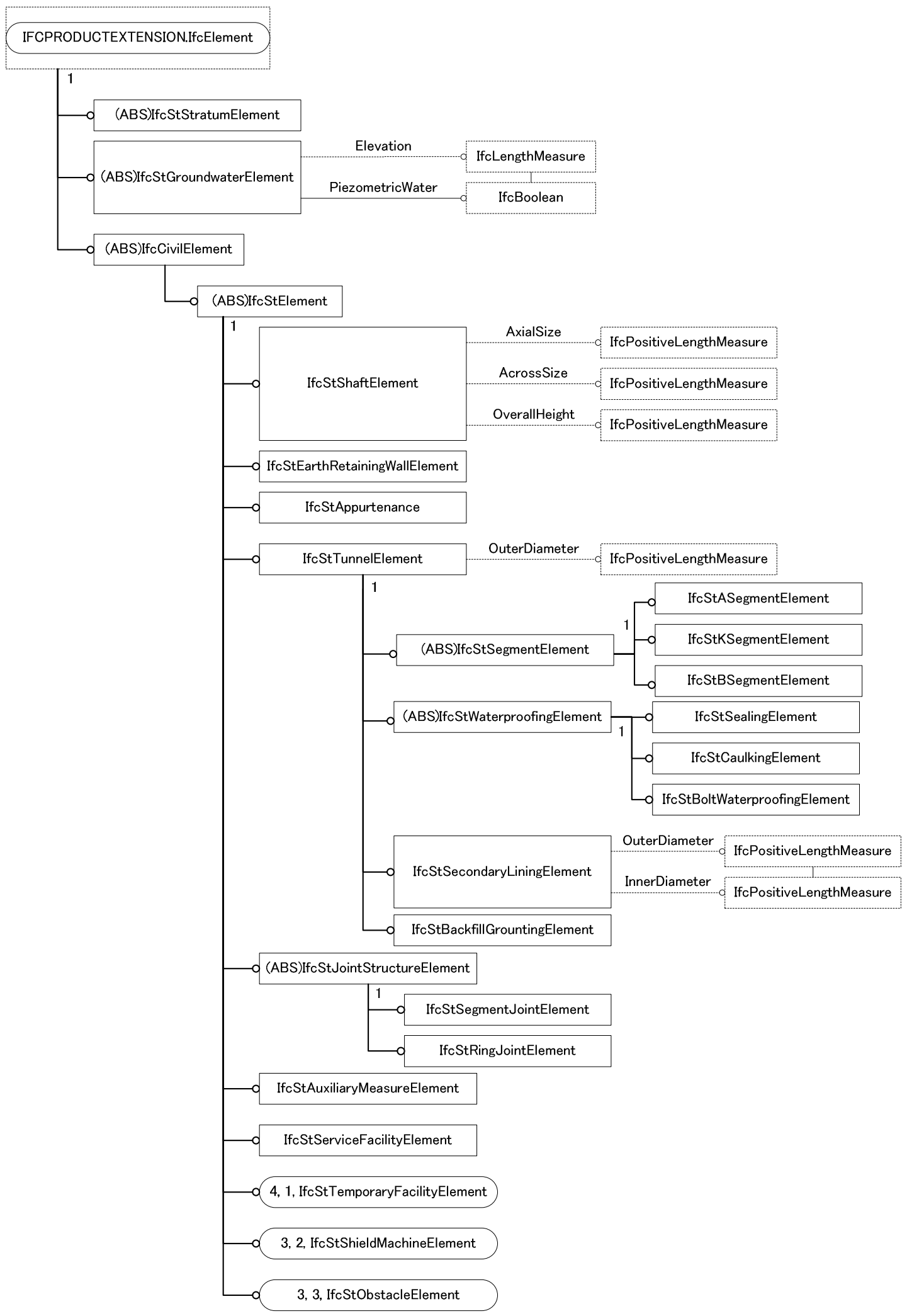

Figure 1 - A part of the product model developed for shield tunnels, IFC-ShieldTunnel 


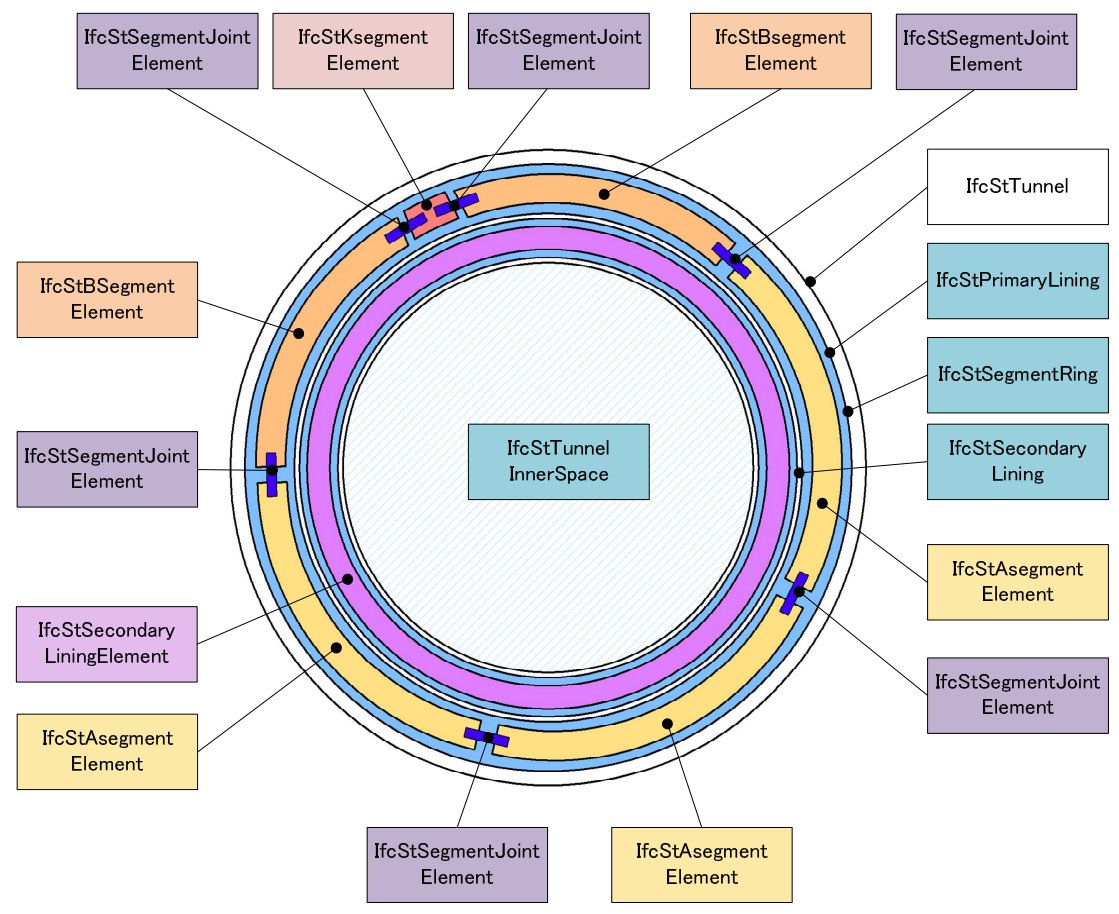

Figure 2 - Section of a shield tunnel and the corresponding entities of IFC-ShieldTunnel

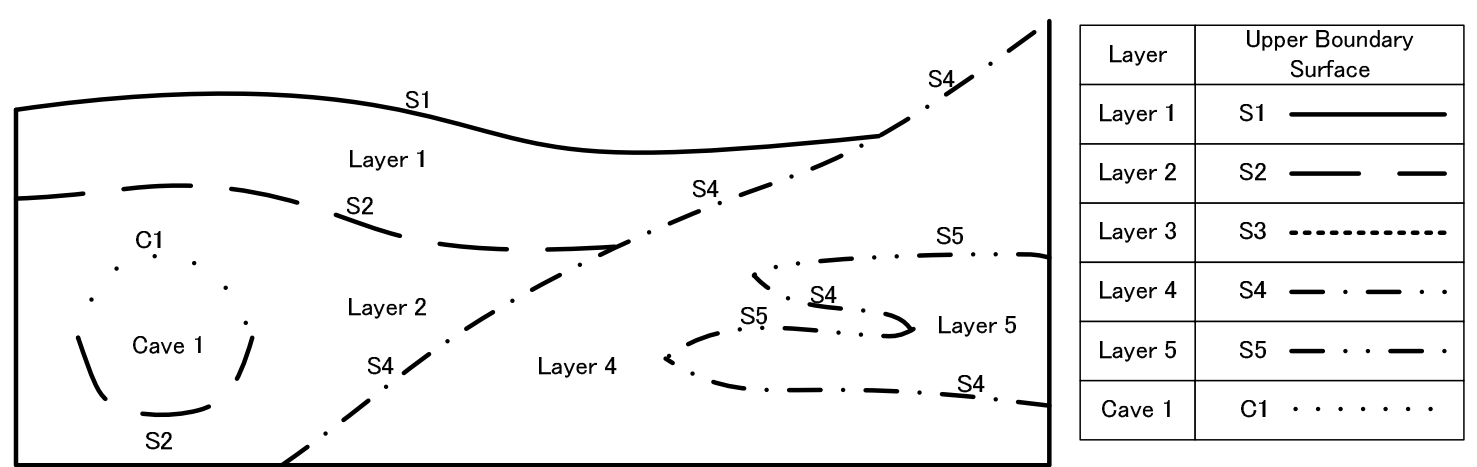

Figure 3 - Representation of soil layers and a cave object in IFC-ShieldTunnel
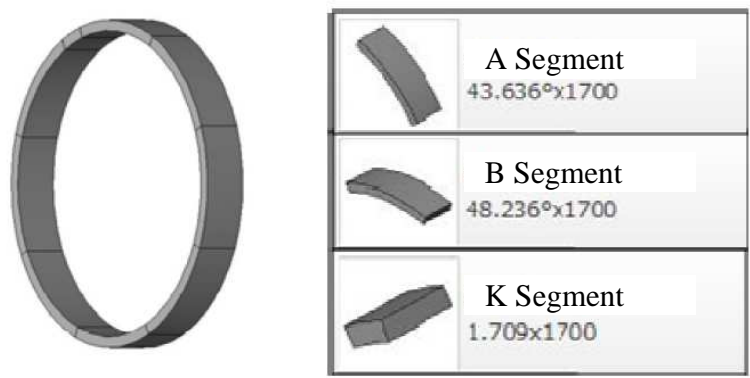

Figure 4-3D models of A, B, and K segments 
Table 1 - Properties of segments

\begin{tabular}{ll}
\hline Parameter & Value \\
\hline Dimensions & \\
\hline H (width) & 1700.0 \\
A (curvature) & $48.236^{\circ}$ \\
\hline Identity data & \\
\hline Keynote & Tunnel member \\
Model & B1 type segment \\
Manufacturer & ABC Corporation \\
Type comments & Structural concrete \\
URL & http://www.abc-c.co.xx \\
Description & Shield tunnel member \\
Assembly code & \\
Type mark & \\
Cost & 100000 yen \\
Omniclass number & 23.25 .30 .21 .14 \\
Omniclass title & Trussed Beams and Joints
\end{tabular}

Since IFC does not yet support shield tunnel segments in IFC-ShieldTunnel, the segment models made using Revit Structure must be represented using existing IFC schema. Thus, the new mapping file shown in Figure 5 was developed, based on the IFC standard. As shown in Figure 5, attributes which are not supported by IFC are "Not Exported." Thus, IfcBuildingElementProxy was used to substitute undefined attributes for existing attributes (Figure 6).

The interoperability of segment data between Revit Structure and Google SketchUp was also examined in this research. A tunnel ring model dataset consisting of segments made with Revit Structure was exported and the exported file was converted with the mapping file and IfcBuildingElementProxy. The converted file was then imported to Google SketchUp and the tunnel ring was drawn successfully (Figure 7).

In addition, at the construction office, a 4D model simulation showing the construction procedure was executed using RevitStructure, and the construction schedule data were exported as an Excel file with segment cost data. Although IFC-ShieldTunnel was not used in this process, the on-site construction engineers were enlightened by this experience and realized the usefulness of using 3D/4D models.

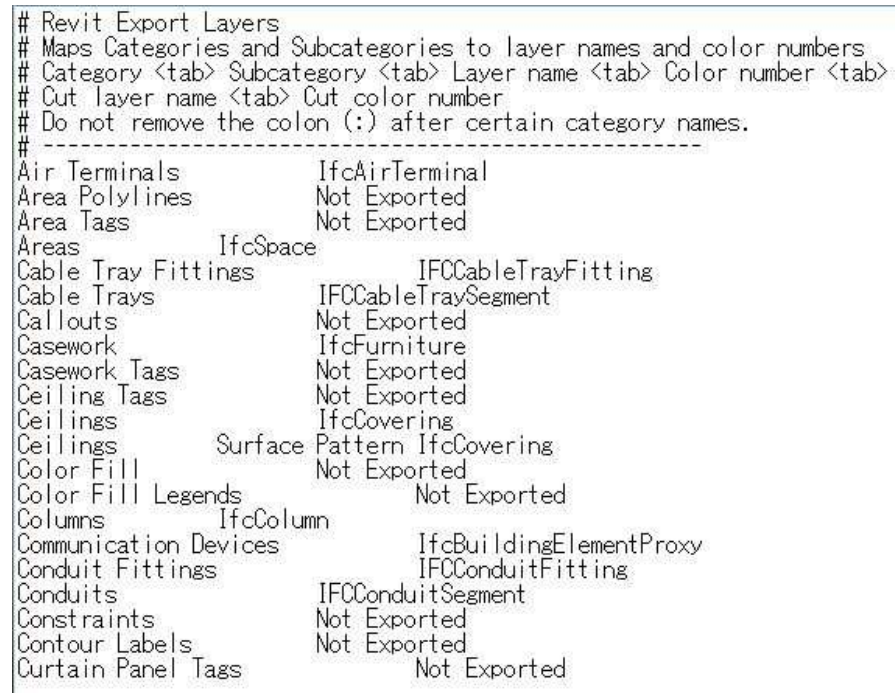

Figure 5 - Mapping file for segments 


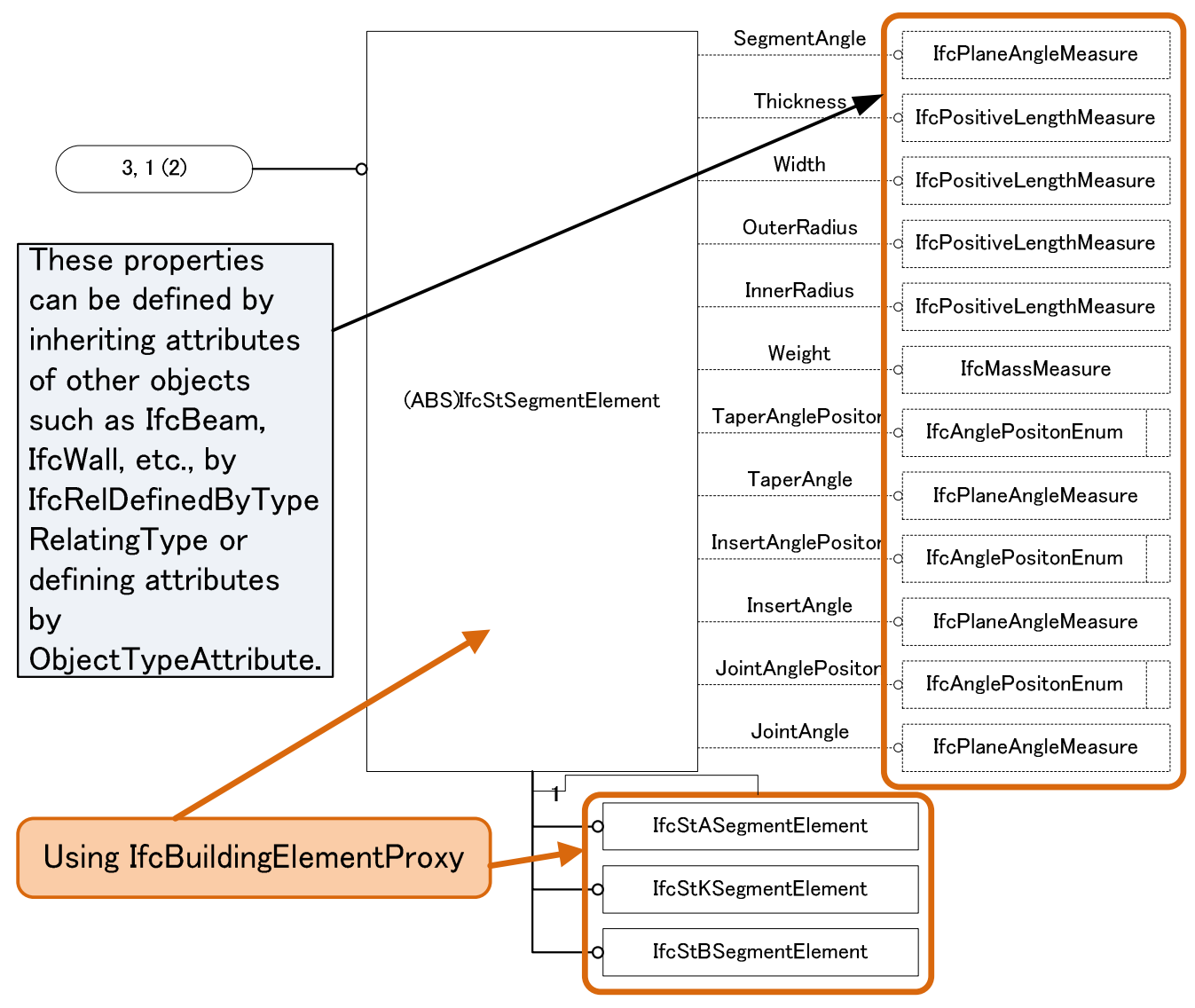

Figure 6 - Substitution of entity and property using IfcBuildingElementProxy

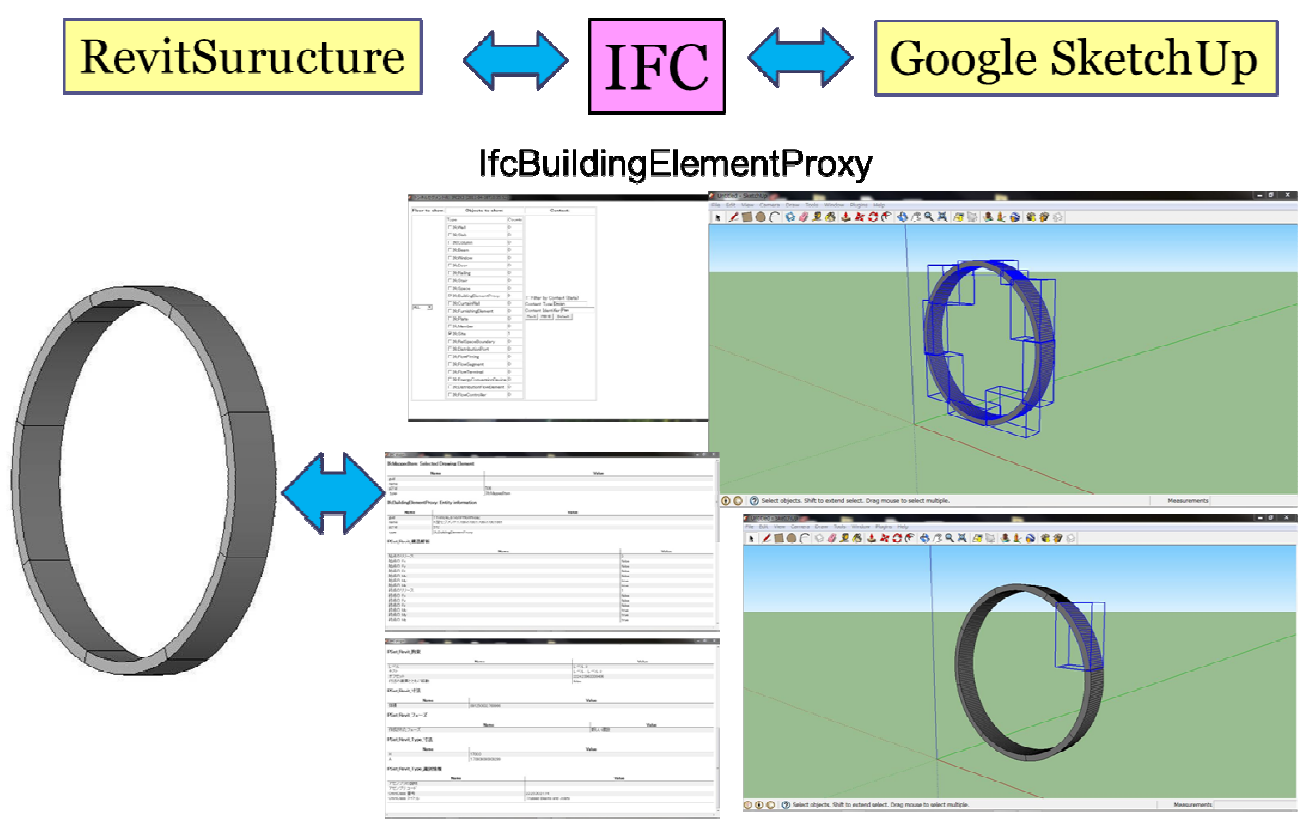

Figure 7 - Segment model data conversion between Revit Structure and Google SketchUp 


\section{CONCLUSIONS}

In this paper, the motivation for and the development process of a product model for shield tunnels, IFC-ShieldTunnel, were described. Then, the application of IFC-ShieldTunnel to an actual construction project was discussed. The development of mapping files and using IfcBuildingElementProxy enabled interoperability between different 3D CAD software packages via IFC-ShieldTunnel for tunnel segment model data.

IFC-ShieldTunnel was completed in 2010 after being modified a few times since 2005 . However, we did not stop developing tunnel product models. In fact, a product model for cut-and-cover tunnels, IFCCut\&CoverTunnel, was developed in 2011 and IFC-MountainTunnel was developed in 2012. These three tunnel product models have been integrated as IFC-Tunnel, which was completed in 2013. We are currently checking the IFC-Tunnel model in collaboration with the OpenINFRA Consortium of buildingSMART International.

\section{REFERENCES}

buildingSMART (2013). buildingSMART Standards. Retrieved from the website: http://www.buildingsmart-tech.org

Eastman, C. M. (1999). Building product models: computer environments supporting design and construction. Boca Raton: CRC Press.

Furuya, H. \& Fujiyama, T. (2011). Development of soil stiffness evaluation equipment, Proceedings of the 28 International Symposium on Automation and Robotics in Construction, Soul, Korea, pp. 337342 .

Yabuki, N. (2008). Representation of caves in a shield tunnel product model, Proc. of the 7th European Conference on Product and Process Modelling, pp.545-550.

Yabuki, N., Azumaya, Y., Akiyama, M., Kawanai, Y., and Miya, T. (2007). Fundamental study on development of a shield tunnel product model. Journal of applied computing in civil engineering 16: in Japanese, pp.261-268.

Yabuki, N., Lebegue, E., Gual, J., Shitani, T., and Li, Z. (2006). International collaboration for developing the bridge product model "IFC-BRIDGE." Proceedings of the joint international conference on computing and decision making in civil and building engineering, pp.1927-1936. 